\title{
Género y sexualidad: consideraciones contemporáneas a partir de una reflexión en torno a la transexualidad y los estados intersexuales.
}

\section{Gender and sexuality: contemporary considera- tions from a reflexion over transexuality and intersexuality states.}

RESUMEN: En el texto se hace un acercamiento a las teorías contemporáneas del género a partir del análisis del caso de la transexualidad y la intersexualidad. Se revisa tanto de los conceptos actuales desde la perspectiva biomédica como de la contestación que se hace desde el campo de las ciencias sociales.

PALABRAS CLAVES: género, identidad, teoría queer, transexualidad, intersexualidad, transgénero.
ABSTRACT: The text gives an approximation to the gender contemporary theories from the analysis of transexuality and intersexuality. Gives a review as the current concepts from the biomedical perspective as the answer that is done from the field of the social sciences. KEY WORDS: gender, identity, queer theory, transexuality. Intersexuality, transgender.

\section{Introducción}

La presencia cada vez más frecuente de personas categorizadas como transexuales en los ambientes clínicos, hospitales y consultas de todo tipo, supone un reto profesional y ético. El avance de las técnicas quirúrgicas y de la moderna farmacología esteroidea, la presión de diversos sectores de la sociedad civil, a favor o en contra del modelo binario, así como las recientes leyes aprobadas en el parlamento español han colocado a la sexualidad, y específicamente la identidad sexual humana en el punto de mira.

Aunque se ha consolidado una responsabilidad ética ligada a la clínica, que incluye la cuestión del género, lo cierto es que existe cierta confusión entre los profesionales, tanto sobre los conceptos que manejamos cuando hablamos de todo esto: sexo, identidad sexual, orientación del deseo, género y práctica sexual; cuanto del manejo práctico de situaciones cotidianas en las que se ven envueltas estas personas. Con frecuencia no sabemos como dirigirnos hacia ellas, a veces se las trata poco respetuosamente y por supuesto nos asombramos, por ejemplo, cuando debemos ingresar a una persona que tiene pene en una cama de mujer.

Todas estas cuestiones producen paradojas interesantes para la reflexión y han estimulado durante años nuestro interés por estas cuestiones. A medida 
que hemos profundizado en el conocimiento de la sexualidad, a medida que nos hemos entrevistado con más personas para hablar de estos temas y de sus experiencias en torno a ellas, las certezas iniciales, fruto de la lectura de los textos clásicos y de las clasificaciones del canon psiquiátrico, fueron transformándose en dudas cada vez más apasionantes. Por otro lado, en el campo de las ciencias sociales y en el seno de la sociedad civil, los discursos de la clínica se ven cada vez más contestados.

En el texto que presentamos a continuación expondremos las paradojas desarrolladas a partir del cruce entre las prácticas médicas y retóricas clínicas y la experiencia de los protagonistas, en mayor o menor medida atravesada por discursos provenientes del campo de las ciencias sociales y humanas. En una primera parte exponemos, sin pretender ser exhaustivos, los aspectos biológicos que sustentarían la representación del sexo como elemento binario para ello partimos del análisis de los conceptos de transexualidad y de intersexualidad. Aunque muchos de estos conceptos han sido cuestionados desde la bioquímica y la genética modernas, su persistencia indica que parecen resistir el embate. En la segunda parte, realizamos un recorrido por las diferentes teorías que desde las ciencias sociales han aportado complejidad a todo este debate. A modo de conclusión, intentamos mostrar lo provechoso de este desplazamiento para la clínica moderna apuntando el concepto de erosdiversidad como posible salida a la trampa binaria de la representación de la sexualidad contemporánea.

\section{Desarrollo sexual, estado de la cuestión}

Empecemos con dos referencias: en un desarrollo normal, hacia la sexta semana de gestación, las gónadas primitivas comienzan a organizarse, pero aún son bipotenciales, es decir pueden dar lugar a testículo u ovarios. Esto depende de la presencia o ausencia del cromosoma Y, en especial de una parte del cromosoma Y llamado antígeno H-Y que, alrededor de la octava semana, inicia la diferenciación testicular, coincidiendo con la secreción de testosterona. En ausencia de dicho cromosoma Y, la diferenciación ocurre a ovarios, sólo que un poco más tarde (duodécima semana). El que los genitales internos o externos se diferencien depende de la presencia, o ausencia, de andrógenos circulantes. Cuando hay una cantidad insuficiente, o los tejidos no responden a ellos, los genitales del feto se diferencian femeninamente, independientemente de la dotación cromosómica e incluso de la dotación gonadal ${ }^{1}$.

\footnotetext{
1 Este argumento está bien desarrollado en numerosos textos recomendamos especialmente Masters, W.H.,Johnson,V.E.,Kolodny,R.C: La sexualidad humana. Grijalbo. Barcelona.1987.
} 
ORIGINALES Y REVISIONES

“...el proceso de diferenciación sexual se puede ver alterado y no seguir el programa preferido por la naturaleza"i.

Las dos citas anteriores, extraídas de sendos textos clásicos de la psiquiatría y de la sexología dejan claro la posición actual de la ciencia con respecto de la diferenciación sexual: las posibilidades son variadas pero solo uno es el programa preferido de la naturaleza. Bajo esta idea subyacen dos consideraciones, en primer lugar podemos encontrar que existe algo, que es externo a las personas que es "la naturaleza" y en segundo lugar que esa naturaleza tiene un plan. Lo interesante en este caso es averiguar como se construye el concepto de naturaleza a lo largo de la historia y como el programa de la modernidad establece esta categoría como algo central, tan central como lo fue Dios en el medievo ${ }^{2}$.

El desarrollo de la biología ha permitido conocer que las cosas no son tan claras como en principio parecen. En efecto, se sabe que, más que de sexos en términos absolutos, podemos hablar de niveles de sexuación. Estos niveles se alcanzan en las diferentes especies que componen la biodiversidad de diferentes formas y en las especies superiores componen un cuadro mucho más rico en matices que la simple dualidad macho/hembra, un cuadro que nos atrevemos a calificar de erosdiversidad.

En este sentido, en los humanos se alcanzarían, al menos, los siguientes niveles: cromosómico, hormonal, anatómico, psicológico y social. Aunque es ambicioso en exceso analizar todos esos niveles y nos conformamos con esbozar algunas de las teorías que sobre los niveles psicológico y social se han planteado contemporáneamente, permítasenos aunque solo sea dar algunas pinceladas sobre los niveles corporales.

A nivel cromosómico, como se ha dicho, se entiende que XX corresponde a mujer, XY corresponde a hombre. Pero la cosa no es tan sencilla: los estados intersexuales suponen un reto para la definición de lo que es hombre y mujer.

Durante siglos fueron los sacerdotes o los jueces los que "interpretaban" el deseo de "la naturaleza" o de Dios para asignar correctamente un sexo. Hay personas con cromosomas $\mathrm{xx}, \mathrm{xy}, \mathrm{x} 0, \mathrm{xxy}, \mathrm{y}$ en todas esas categorías los fenotipos pueden variar entre lo que entendemos por hombre y lo que entendemos por mujer pasando por una amplia variedad de configuraciones corporales alternativas ${ }^{3}$. Los jueces se veían obligados a decidir en litigios sobre sexo dado que hombres y mujeres tenían diferentes derechos en cuanto a la herencia, el sufragio, etc. En la historia judicial de los siglos XVI al XIX, existen numerosos ejemplos al res-

\footnotetext{
${ }^{2}$ el debate en torno a la naturaleza excede el propósito de este texto si quien lee desea avanzar en este camino recomendamos los debates entre Donna Haraway y Bandana Siva y también las aportaciones de los últimos trabajos de Castilla Vallejo: Castilla Vallejo, J.L. et al. Postdesarrollo y Naturaleza. Estudio sobre la sierra gorda de Querétaro (Mexico). Ed. Porrúa. Mexico. 2008 y La Modernidad contra el agua. Saberes sometidos y gestión de la demanda. El caso de la reserva de la biosfera del hierro (en prensa).
} 
pecto algunos de ellos célebres ${ }^{\text {ii }}$. De hecho en los recientes Juegos Olímpicos de Beijing se habrán tomado muestras celulares de cada deportista para asegurar su estatus sexual y no siempre ofrecen el resultado que se espera ${ }^{4}$.

Posteriormente, fueron los médicos los que tomaron el papel intentando definir dónde reside el verdadero sexo. Para ello han mirado insistentemente en el cuerpo y en el cerebro. Se ha medido, pesado, cortado hasta la saciedad intentando encontrar algo que definitivamente nos de datos fehacientes de dónde se encuentra dicho verdadero sexo. La ciencia intenta descubrir por qué los hombres y las mujeres piensan de manera diferente, pero ¿es que acaso piensan diferente? Muchos autores contemporáneos han observado que más que interpretar la naturaleza para descubrir leyes que puedan ser aplicables al mundo social los, científicos se valen de verdades que se extraen de nuestras relaciones sociales para estructurar, leer e interpretar la naturalezaiii.

Un ejemplo clarificador, el estudio que durante la década de los 90 se centró en las diferencias entre los cuerpos callosos de varones y mujeres. En el siglo XIX el cuerpo calloso también estuvo implicado en el estudio de las diferencias de índole racial, algo que se considera políticamente incorrecto en nuestros días. Este complicado sistema, auténtica central telefónica que conecta nuestros dos hemisferios cerebrales, es tan complejo en su morfología que disecarlo para medirlo es prácticamente imposible, por eso todos los estudios se basan en proyecciones bidimensionales de un corte sagital, esto es, una técnica indirecta, o bien mediante medidas de imágenes de RMN, también indirectas. En la actualidad casi nadie piensa que la superficie de dicha proyección sea diferente entre los diferentes sexos, tampoco en las diferentes razas, por lo que algunos grupos de investigación comenzaron a subdividir el cuerpo calloso en diferentes partes de forma arbitraria y aparecieron diferencias que se interpretaron de forma diversa por los diferentes grupos. Por ejemplo el llamado esplenio del cuerpo calloso es considerado por muchos sexodependiente y por otros no, finalmente un metanálisis ${ }^{\text {iv }}$ determinó que a medida que se aumentaba la muestra en los estudios las diferencias se disolvían hasta desaparecer. Lo importante para nuestro trabajo es cómo nuestra domesticada observación de los hechos los hace coincidir con nuestras ideas previas que raramente son cuestionadas.

Algo similar podemos decir del aspecto hormonal. A finales del siglo XIX los investigadores comienzan a estudiar las sustancias químicas contenidas en los tejidos y en las glándulas sexuales mediante técnicas de corte y de transplante.

\footnotetext{
${ }^{3}$ La mayoría de los autores preferirán usar el término intermedio, pero ello implica considerar que sólo los extremos son fisiológicos mientras que todo lo demás entra en el rango de lo patológico.

${ }^{4}$ El caso de la deportista española María Patiño, a pesar ser anatómicamente una mujer y vivir toda su vida como tal, descubre que cuenta con dotación cromosómica XY al llegar a los juegos olímpicos de 1988 acabando de un plumazo con años de entrenamiento, sus esperanzas, su trabajo como deportista de élite... (citado en Fausto-Sterling, A. cuerpos sexuados, Ed. Melusina, Barcelona 2006.
} 
ORIGINALES Y REVISIONES

Los científicos diseñaron sus experimentos, interpretaron los resultados y comprendieron el funcionamiento de estas sustancias a través de una matriz cultural previa que establecía que existían dos sexos antagónicos por tanto: deberían existir machos que sólo producen hormonas masculinas supresoras del desarrollo femenino aún en presencia de hormonas femeninas y por otro lado deberían existir hembras que producen sólo hormonas femeninas supresoras del desarrollo masculino aun en presencia de hormonas masculinas ${ }^{\mathrm{v}}$.

Durante décadas se mantuvo la hipótesis de las esferas sexuales antagónicas, siendo de hecho la que actualmente continua como referencia para la mayoría de la población no formada en bioquímica (incluidos muchos profesionales de la salud). Pero una vez más los hechos se empeñan en porfiar con nuestras concepciones culturales y muestra que estas sustancias, las hormonas, se encuentran en todos los cuerpos en proporciones muy variables de uno a otro individuo. Una vez más son las consideraciones apriorísticas las que definen los efectos de determinada sustancia como virilizante o feminizante. Todo esto en un contexto social, las décadas de los 20 y 30, de cambio en las concepciones del género en occidente. Ya en 1936 John Freud, un bioquímico holandés que investigaba el mundo de las hormonas sugirió abandonar el uso del concepto de hormona sexual a favor del de biocatalizadores, argumentando que diversos órganos producen esteroides y muchos otros responden ante ellos. En determinadas condiciones favorecen el desarrollo sexual porque son hormonas que favorecen el desarrollo de los tejidos. Son poderosas sustancias que catalizan el crecimiento de los tejidos, de prácticamente todos los tejidos, por supuesto también de los tejidos que intervienen en la respuesta sexual ${ }^{\mathrm{vi}}$.

La gestión médica de dichos tejidos también se ve atravesada por nuestras teorías sobre el sexo y el género. Esto se ve marcadamente en el estudio de los estados intersexuales, que es como la ciencia medica llama a las personas cuyos cuerpos no cumplen el "plan preferido de la naturaleza". Lo establecido en los diferentes tratados de cirugía es que las personas con órganos sexuales indiferenciados o ambiguos deben ser reasignadas quirúrgicamente lo más precozmente posible so pena de llevar una vida sumamente desgraciada, lo que desde luego no está corroborado por la investigación, por lo demás escasa. (Figura 1)

Sí existe noticia, a lo largo de la historia, de personas que han vivido en un sexo o en otro, cumpliendo razonablemente no solo con los aspectos de rol sino con los aspectos físicos de cada caso. Sabiendo que los genitales humanos proceden de estructuras indiferenciadas que se desarrollan en un sentido u otro nos podemos preguntar a partir de qué medida un clítoris grande se convierte en un pene pequeño. Pues bien, esta escala existe, falómetro la denomina Fausto-Sterling, y en función de ella (ver figura 1) se deciden cirugías extraordinariamente agresivas que acaban, la mayoría de las veces, con la opción de una sexualidad 
satisfactoria para garantizar una socialización adecuada en un medio rígido, que no tolera disidencias corporales. Una cirugía estética para adaptar una variedad corporal posible y legítima a una disciplina político-social limitante, en palabras de la autora.

Por otra parte no todo parece ocurrir prenatalmente, existe un importante debate entre los que opinan que todo se debe a la bioquímica cerebral y a estructuras anatómicas predeterminadas en el mapa genético y los que opinan que hay una influencia de la cultura. Es clásico citar los trabajos de Money sobre la influencia de la crianza en el proceso de reasignación. Aunque su experimento, el célebre caso John/Brenda, a la larga demostró ser un despropósito de funestas consecuencias, desde sus trabajos se admite la existencia de una importante influencia del medio ambiente después del nacimiento ${ }^{5}$ que influiría notablemente en fenómenos como la orientación del deseo sexual o de la identidad sexual ${ }^{\text {vii }}$. Por tanto existirán cuatro funciones independientes: el sexo, entendido como la configuración corporal con dos posibles destinos "normales" de la diferenciación sexual (macho y hembra); la orientación del deseo, es decir qué tipo de personas, o cosas, son las capaces de producir activación sexual en cada cual, (homosexual, heterosexual, parafílico); la identidad sexual que es la firme convicción que se tiene de pertenecer al propio sexo y finalmente estaría, como algo aparte, el género que se refiere a los aspectos suprapersonales, de origen social, lo que se espera de cada cual por tener una determinada forma corporal. Aunque la ciencia médica en general evita hablar del género reduciéndolo a un mero rol, por ser algo más propio de la ciencia social, es un aspecto de la mayor importancia cuando se aborda el tema de la transexualidad.

\section{Transexualidad y sexo psicológico.}

La transexualidad también es un fenómeno antiguo. El caso más antiguo identificable como transexual probablemente sea el rey asirio Sardanapalus, que vestía de mujer y paseaba con sus esposas. Hay muchos más casos: Filo de Judea, durante el Imperio Romano, el caballero Eon, vivió durante 39 años como mujer y se disputaba los amores de Luis XV con Madame Pompadour, Lord Cornbury, primer gobernador colonial de Nueva York, pisó tierra americana vestido de mujer y siguió así hasta el final de su mandato ${ }^{\text {viii. }}$

\footnotetext{
${ }^{5}$ También antes del mismo, por ejemplo la presencia de un ambiente rico en andrógenos en las semanas previas al nacimiento influiría en futuros comportamientos y se ha esbozado como posible causa de homosexualidad si que exista evidencia científica ni de una cosa ni de la otra.
} 
ORIGINALES Y REVISIONES

Sin embargo, el transexualismo, entendido como trastorno aparece en los sistemas de clasificación no antes de 1980, a pesar de que ya desde 1966 Harry Benjamin, en su libro The transexual phenomenon ${ }^{i x}$, había definido los criterios diagnósticos básicos que durante décadas fueron tomados como referencia. Se crearon las primeras clínicas en EEUU, aunque en Marruecos hacía años que funcionaban y se comenzó con los programas de reasignación sexual. Los científicos y terapeutas se dieron cuenta, al valorar la conveniencia de realizar o no el proceso de reasignación, que los individuos analizados se ajustaban muy bien a los criterios del libro de Benjamín, los científicos y terapeutas tardaron una cantidad de tiempo inusitado en darse cuenta de que ese ajuste se debía precisamente a que el libro circulaba entre las comunidades trans desde hacía años ${ }^{\mathrm{x}}$. Más recientemente los dos sistemas de clasificación principales, el Diagnostic Stadistical Manual (DSM) y la Clasificación Internacional de Enfermedades (CIE), establecen sus propios criterios, aunque básicamente similares, para el diagnóstico del trastorno pasando por diversas nomenclaturas, transexualismo, disforia de género y finalmente trastorno de la identidad sexual. Un análisis más preciso nos revela algunas contradicciones y singularidades a tener en cuenta:

El DSM IV-TR ${ }^{\mathrm{xi}}$ considera cuatro criterios para alcanzar el diagnóstico de transexualidad. Quisiéramos empezar por analizar estos criterios (ver tabla 1): el primero establece la identificación con el otro sexo y acota, no solo el deseo de obtener las supuestas ventajas relacionadas con las costumbres culturales, para después pasar a describir las conductas que reconoce el terapeuta, indicativa de dicha identificación, tanto en niños como en adultos y adolescentes. Estos rasgos consisten en una afirmación de pertenecer al otro sexo y demostrarlo mediante determinados gustos, deseo de usar una determinada clase de ropa o ser tratados de una determinada manera que, sin embargo, sólo es posible entender como sujetos a un condicionamiento de índole exclusivamente cultural. El segundo de los criterios se refiere exactamente a lo mismo pero en negativo, es decir se refiere al disgusto de pertenecer al sexo asignado al nacer. (Tabla 1)

La CIE $10^{\text {xii }}$, publicada con posterioridad al DSM IV no añade elementos que mejoren el rendimiento diagnóstico. En ambos casos, a pesar de lo que reza en el título del epígrafe correspondiente, los rasgos y las conductas requeridas son exclusivamente culturales.

Porque el problema, desde este punto de vista no se encuentra en aquellas personas que sienten que algo no encaja entre su cuerpo y su mente. El problema está en el modelo que hace que esas personas sientan que algo no encaja entre su cuerpo y su mente, un modelo que por otra parte se nos presenta como dado, esto es, como anterior a nuestra cultura. Un modelo que define dos sexos "naturales" contrapuestos destinados a desearse cada uno al otro y que otorga unas características a cada uno que son definitorias y excluyentes. 
ORIGINALES Y REVISIONES

\section{Sexo físico versus sexo psicológico (psicosocial) ${ }^{6}$}

El hecho transexual implica la consideración de una doble ontología. Por una parte, estaría el sexo biológico como una expresión física y, por la otra, el sexo psicológico que se expresa a través de una función llamada identidad sexual, que consiste en la convicción que tenemos las personas de pertenecer a un sexo o al otro. Beatriz Preciado, destacada pensadora y activista queer, se refiere y define, con respecto a las estrategias de reasignación sexual en los transexuales, a esta doble ontología: por un lado están los criterios de asignación de sexo, al nacer o prenatalmente, que determinan que un cuerpo es masculino o femenino mediante un modelo de reconocimiento visual, cuyos significantes (genitales, cromosomas u hormonas) se consideran empíricos y científicos (ontología escópica: lo real es lo que se ve) frente a la idea de que existe un sexo psicológico verdadero que es distinto, en el caso de la transexualidad, de aquel que ha sido asignado en el nacimiento, el sentimiento interior de ser un hombre o una mujer, que pertenece a un modelo de lo radicalmente invisible, no representable (una ontología inmaterial: lo real se esconde a los sentidos) $)^{\text {xiii. }}$. Sin embargo, a diferencia de otras convicciones personales, en las que podemos contrastar las experiencias, la propia consideración sexual no es contrastable, esto es, no hay forma de experimentar una "propia concepción sexual" diferente a la que tenemos, incluso no es posible compartir nuestra experiencia íntima de ser o pertenecer a un determinado sexo con personas de ese mismo sexo y por tanto no podemos defender nuestra propia identidad sexual salvo mediante conductas. Las conductas que se esperan de mí por ser de un sexo u otro. Por otra parte sabemos qué se espera de un sexo u otro por el aprendizaje social. En otras palabras la identidad sexual no es algo íntimo o innato, es algo construido y enseñado. Como consecuencia de lo anterior podemos concluir que no hay diferencia entre los conceptos de identidad sexual y de género. Podríamos decir que la identidad sexual es la interiorización del género y que el género es la expresión pública de nuestra identidad sexual. Esta relación entre género y cuerpo ha sido muy debatida en las últimas décadas, sobre todo en el ámbito feminista.

\section{Teoría del género}

Ya Simone de Beauvoir declaró "no se nace mujer sino se llega a serlo"xiv, dándose cuenta de que la biología no explicaba el carácter de "el otro" de la mu-

\footnotetext{
${ }^{6}$ La LEY 3/2007, de 15 de marzo, reguladora de la rectificación registral de la mención relativa al sexo de las personas ,establece una nomenclatura diferente a la establecida en la literatura científica, habla de sexo morfológico o género fisiológico frente a identidad de género o sexo psicosocial. Sin embargo clínicamente se establece una diferencia entre la identidad sexual y el género.
} 
ORIGINALES Y REVISIONES

jer. No obstante esta visión establece también la existencia de "un uno", el varón, que ejerce su dominación en primera persona. Otra autora, Teresa de Laurentis, abunda en este argumento de la construcción social del género, planteándolo más bien como un cruce de discursos que proceden de los dispositivos disciplinarios, entendiendo disciplinario en el sentido foucaultiano del término y un tanto opuesto al soberanismo ${ }^{\mathrm{xv}}$ que reivindica Beauvoire. Entre estos dispositivos y sin pretender ser exhaustivos, estarían: familia, religión, escuela, medicina, mass media pero también el cine, los seriales televisivos, la literatura, el arte y, como vimos, la ciencia. Frente al binomio victima-opresor De Laurentis establece el concepto de tecnología(s) del género para definir el conjunto de ritos, mensajes, discursos y prácticas que tienen como resultado la conformación del género. Estas tecnologías se aplican a todas las personas independientemente de en qué sexo hayan sido educadas ${ }^{\mathrm{xvi}}$, se aplican de manera anónima, es decir no hay responsable, ni vigilante nominado, al contrario es el conjunto de las personas las que ejercen ese control, mas allá, la importancia de la autocensura es vital en la perspectiva de esta autora, la autovigilancia y el autocastigo. Sin embargo la crítica de la dicotomía sexo-género, permanece aún asentada en la firme convicción de la dicotomía de los cuerpos, lugar donde convergen todas las vías.

La definición del "sujeto histórico" en el caso del feminismo no escapa a esta contradicción si las mujeres son un grupo diferenciado qué criterios habrá que seguir para incluir a alguien entre sus filas, hasta que punto la definición de esos criterios no genera nueva división y nueva desigualdad ${ }^{\mathrm{xvii}}$. Y no estamos hablando desde argumentos conservadores y patriarcales, hablamos de por ejemplo, cómo se le puede impedir la entrada a un encuentro feminista a una mujer transexual ${ }^{\text {xviii }}$ y también hablamos de la denuncia que, desde el campo homosexual, se hace. Precisamente desde ese campo, destacamos a Monique Wittigix, su afirmación "las lesbianas no somos mujeres" tiene el sentido de que no se identifica con esos parámetros dicotómicos hombre-mujer complementarios y desde luego denuncia el heterocentrismo que, según su criterio, subyace bajo el argumento feminista. Por tanto se trataría de equilibrar al menos tres esquemas dicotómicos: dicotomía de los cuerpos, dicotomía de los géneros, dicotomía de los deseos (homo/hetero).

La pensadora estadounidense Judith Butler, apoyándose en una crítica de la teoría lacaniana, de la que se siente heredera, en las aportaciones del feminismo y en especial del feminismo lesbiano y en la genealogía de Michel Foucault, ha elaborado una revolucionaria manera de abordar las tres dicotomías anteriormente descritas.

\section{Teoría performativa del género}

Para Butler el género es la matriz cultural que permite que exista, que habite la idea de un sexo natural o una naturaleza sexuada. Esto produce el efecto, 
la ficción, de que esa naturaleza sexuada es anterior a la acción de la cultura. De esa manera pensamos que existe un sexo natural sobre el que actuaría la cultura y como consecuencia se produciría el género, siendo esta la visión clásica tanto de las corrientes feministas como de la propia ciencia. Según Butler, lo que ocurriría sería lo contrario. Como consecuencia de que tenemos una cultura que piensa en términos de género se ha hecho una explicación sexuada de la naturaleza. Cómo se produce esto es lo que estudia a lo largo de las últimas décadas en sus distintos trabajos algunos de los cuales han sido y son realmente influyentes.

La anticipación de un objeto lo genera, algo que conocemos bien en la clínica como por ejemplo cuando vemos que la anticipación de un síntoma, por ejemplo la ansiedad, es precisamente lo que la genera provocado la ilusión de que el objeto creado se encuentra en el origen del miedo cuando en realidad es su consecuencia. Butler establece lo mismo respecto del género: una expectativa previa de que los géneros son dos y antagónicos y que los sexos son dos y antagónicos produce el efecto de que veamos a los sexos dos y antagónicos. Posteriormente esto se mantiene y se reproduce mediante la repetición ceremonial y difusa de una sucesión de actos simbólicos y cotidianos, actos de habla y acciones sociales ceremoniales que se comportan como performances ${ }^{\mathrm{xx}}$, es decir, como eventos que generan "realidad". Estas tres ideas que acabamos de esbozar son lo que constituye el esquema básico de la teoría performativa del género una de las mas influyentes y, lógicamente, más controvertida.

Un acto performativo es un acto, generalmente verbal aunque no sólo verbal, que al ser ejecutado genera una realidad nueva. Podríamos poner algunos ejemplos: "es una niña", "les declaro marido y mujer", "eres un marica", etc. En el caso del género, Butler lo considera performativo porque construye la realidad a la que se supone que representa. Por tanto, el género no seria un elemento o un evento que se constituye de una manera estable, un suceso, sino más bien una acción: un hacer, pero que no es un hacer por parte de algo que pueda supuestamente ser anterior a ese género. Coincidiendo con Nietzche cuando afirma "no hay ningún ser detrás del hacer, del actuar, del devenir"xxi para Butler no existe ninguna esencia que se exprese en el género, puesto que no es un hecho sino un conjunto de actos, un devenir. Más que una verdad psicológica el género (y por tanto la identidad) es una práctica: una práctica del discurso, una practica del cuerpo y una práctica de las relaciones y a través de ese hacer es como las personas adquieren inteligibilidad en el medio social. Debemos darnos cuenta que en nuestra sociedad hasta que no sabemos el sexo de una criatura, es como si no supiéramos quienes son realmente, lo que se nota claramente en el desasosiego que nos produce a los profesionales referirnos a estas personas, no sabemos si decir la, si decir lo... 
ORIGINALES Y REVISIONES

\section{Posibilidades para el cambio}

El análisis de Butler, es un análisis feminista, heredero entre otras de Beauvoir, Teresa de Laurentis y Wittig, y puede verse como pesimista y desesperanzado. ¿Qué nos queda pobres personajes presos de nuestros propios fantasmas e ilusiones? Butler plantea que el desvelamiento del mecanismo libera la posibilidad de su destrucción. Saber que la repetición de estas categorías no se puede realizar de manera pura y en las sucesivas repeticiones ocurren desplazamientos que generan fallas, también provocados por el empecinamiento de la gente en desafiar las convenciones, Butler considera esas fallas como el lugar donde se ubica la posibilidad de la trasgresión y de la subversión de la trampa. Lo que queda pues, propone, es desvelar los mecanismos y también queda apropiarse de ellos. Algo que está ocurriendo ya desde hace décadas en muchos lugares, principalmente ciudades de la costa este de EEUU, donde la transgresión se ha convertido en una importante arma política. Esta transgresión en realidad es anterior a la teoría descrita, Butler acude a los márgenes, a aquellos lugares donde se producen estas fallas del discurso, donde se vuelve problematizado y encuentra una importante fuente en los movimientos y las prácticas sexuales alternativas. Un ejemplo es el fenómeno drag, donde se da un cruce de conceptos y de psiquismos. Un auténtico rizo conceptual. Mi apariencia exterior es femenina pero mi esencia interior es masculina y al mismo tiempo mi apariencia externa es masculina (soy una imitación) pero mi esencia interior "yo" es femenina. Sin embargo lo que interesa a Judith Butler es comprender que al imitar al género lo que copian las drags no es un original sino que imitan una copia, una copia misma. Al imitar el género se manifiesta implícitamente la estructura imitativa del género en sí. El género (y por tanto la identidad sexual) es siempre imitación ${ }^{\mathrm{xxii}}$.

Beatriz Preciado va un poco más allá y plantea que la certeza de ser hombre o mujer es una ficción somática y política producida por un conjunto de tecnologías, en el sentido que le daba Teresa de Laurentis, es decir: tecnologías de domesticación corporal tanto con medios farmacológicos como con medios audiovisuales, de tal manera que se produce una fijación y una delimitación de nuestras posibilidades. Un importante efecto de esa delimitación y fijación es la producción de un saber sobre sí mismo, de un sentido del yo sexual que aparece como una realidad emocional evidente a la conciencia ${ }^{x x i i i}$. Las personas saben que "Soy hombre-mujer-hetero-homo-trans..." Tienen la conciencia de ser algo que no es más que una ficción, dejando fuera de la comprensión social múltiples realidades vigentes.

Esa delimitación a su vez, se comporta como un cordón que separa lo normal de lo anormal, lo correcto de lo incorrecto Preciado insiste en la creación de diferentes ontologías a propósito de la cuestión trans: la del cuerpo visible frente 
al sexo psicológico ya vista pero también la del bio frente al tecno sexo, en el que habría por un lado biohombres y biomujeres entendidas como las que conservan su "sexo original" y por otro lado tecnohombres y tecnomujeres que serían las que presentan un sexo construido en un proceso técnico ${ }^{\text {xxiv }}$. Finalmente, podemos incluir una diferenciación generada a partir de la ley de identidad de género, ley que no obliga a completar un proceso de transexualización mediante la cirugía para que pueda modificarse el sexo en el registro civil, es más la obligatoriedad de recibir un tratamiento médico de al menos dos años de duración, se entiende que hormonal, no es tampoco obligatorio si existen razones de salud física que lo desaconsejen. El resultado inmediato de todo esto es indudablemente la existencia de mujeres con pene y hombres con vulva ${ }^{\mathrm{xxv}}$.

Pero, si todo esto es así, entonces dónde quedan los criterios diagnósticos DSM de la transexualidad. Cuales son esas características que definen un hombre y una mujer como tales. En qué consiste exactamente ser hombre y ser mujer. Qué significan las reasignaciones y cómo influyen en nuestra concepción de la realidad. Ya comentamos que la reasignación sexual pivota en torno a la existencia de dos modelos incompatibles y opuestos de reconocimiento por un lado el visual y por otro el psicológico. Pero si tenemos claro que este último sólo es una práctica, un conjunto de actos. ¿qué necesidad hay de que una persona se someta a intervenciones farmacológicas y a intervenciones quirúrgicas que en la mayoría de los casos acabarán de manera irreversible con la posibilidad de placer sexual? ¿Por qué no se puede existir y comportarse, expresando la forma de sentirnos personas sexuadas/les como nos complazca mejor?

Durante décadas la lucha de la comunidad transexual por conseguir el respeto y la consideración por parte de la sociedad, la lucha por conseguir que los tratamientos de reasignación estén disponibles en condiciones de seguridad ${ }^{7}$, posteriormente fueran incluidos dentro de las prestaciones de las distintas formas de seguro social de los países y posteriormente que su condición fuera aceptada legalmente ha pasado, paradójicamente, por su identificación como enfermedad, a diferencia de otras "minorías" sexuales que siempre se revelaron contra la medicalización de sus opciones y sus placeres.

Como respuesta a esto, tanto desde los colectivos intersexuales, que se resisten a que se les obligue a decidir entre uno u otro sexo, como del propio campo trans se está produciendo la reacción. Algunas organizaciones de transexuales han comenzado a promover la idea del transgenerismo a partir de la critica al transexual tradicional: Son personas que pueden tomar hormonas pero no se ope-

\footnotetext{
${ }^{7}$ Durante décadas los transexuales españoles viajaban a Casablanca donde se realizaban las operaciones en condiciones dudosas y con escaso seguimiento pre y posquirúrgico, en comparación con lo que se hace actualmente en el Estado Español.
} 
ORIGINALES Y REVISIONES

ran, o visten conforme a su opción de género (independientemente de cómo se configure su cuerpo) y no toman hormonas ni se operan, o bien visten de hombre o mujer según el día o el momento o adoptan roles diferentes en diferentes contextos. Algunas de estas personas se mantienen permanentemente en un estado de ambigüedad sin optar. Para el transexual, digamos tradicional estas personas estarían en transición. Cuando el objetivo es alcanzar el ideal corporal establecido, la alternativa es transitar o conformarse. Frente a esta visión el transgenerismo plantea que los transexuales tradicionales lo único que hacen con su deseo de acercarse al rol tradicional del sexo opuesto es apuntalar toda esta economía del sistema sexo-genero y paradójicamente como consecuencia de eso un refuerzo de los mecanismos sociales que están en el origen mismo de la llamada disforia ${ }^{8}$. Desde estas posiciones, como se adelantó antes lo que habría que hacer más bien, es subvertirlos, entre otras formas mediante la apropiación y el uso adulterado de las reglas de género. Estos y otros numerosos movimientos sociales reivindican cada vez más el no querer encasillarse y reivindican sus legítimas rarezas. Algunos de estos movimientos han comenzado a apropiarse incluso de la injuria, el insulto, entendiendo a estos cómo el origen de su identidad ${ }^{\mathrm{xxvi}}$, No es extraño pues que se hayan denominado movimientos Queer ${ }^{9}$ y la teoría que se ha producido en torno a ellos Teoría Queer.

Recientemente los medios de comunicación se han hecho eco del caso Beatie. Thomas Beatie, de 34 años, apareció en el show de Oprah Winfrey con una prominente barriga, correspondiente a sus ya más de cinco meses de embarazo, sin abandonar su apariencia masculina, pues lucía cabello engominado pelo corto, y una barba recortada.

Nació mujer, Tracy Langordino, en su juventud hizo sus pinitos como modelo, e incluso llegó a la final de un concurso de belleza en Hawai.

Hace cinco años, una vez la ley ya reconoció a Beatie como un hombre, se casó con Nancy Roberts, después de cinco años de noviazgo. Nancy y Thomas tomaron la decisión de tener un hijo juntos poco después de la boda. Puesto que no podía concebir por haberse sometido en el pasado a una histerectomía a causa

\footnotetext{
8 Una economía política ciertamente que establece que por un lado existen órganos del cuerpo y partes del cuerpo que nos pertenecen por ejemplo el pelo, todo el pelo del cuerpo nosotros podemos hacer con el lo que queramos: pintarlo, cortarlo de diversas maneras, etc. Aquí también estaría la nariz, si tengo dinero me voy a un cirujano que me enseña un catálogo de narices elijo una y me la hacen. Con otras partes podemos hacer según que modificaciones. Por ejemplo en las mamas podemos poner, si tenemos dinero podemos ir a un cirujano y decirle que nos ponga silicona y prácticamente no hay límite para la silicona que se puede poner, pero quitar es otra cosa pedir una mastectomía electiva es algo implanteable, requiere un diagnóstico de cáncer o un diagnóstico de transexualidad. Lo mismo pasa con los genitales si deseara una vaginoplastia necesitaría un diagnóstico y muchas explicaciones... es como si esas partes del cuerpo fueran de una propiedad diferente a yo mismo.

9 Aunque la palabra significa invertido, concepto que en español es un eufemismo, en ingles se trata de un insulto equivalente a marica. Muchos de los primeros textos en español incluyen traducciones como teoría torcida, pero actualmente o bien se deja sin traducir o se traduce de manera general como teoría marica.
} 
de una enfermedad, debía ser Thomas quien quedara embarazado. "Si Nancy pudiera quedarse embarazada, yo no estaría haciendo esto",

Hay muchos otros casos, me parece especialmente fascinante el de Jane / John y Mary, citado en el libro de Fausto-Sterling ${ }^{\text {xxvii }}$, Jane, nacida varón, cercana a los cuarenta y que continua viviendo con su esposa Mary, con quien se había casado cuando era todavía John. Jane toma hormonas pero este tratamiento no ha menoscabado su capacidad de tener erecciones y penetrar a su esposa.

Desde su perspectiva, Jane tiene una relación lésbica con Mary. Pero también usa su pene para el placer. Mary no se identifica como lesbiana aunque siente amor y deseo hacia Jane, la primera persona de quien se enamoró, aunque haya cambiado físicamente. Mary se considera heterosexual.

\section{Conclusiones.}

Hemos dado tan sólo unas pinceladas del pensamiento contemporáneo sobre el género y el sexo. Muchas veces se plantea que este tipo de análisis "postmoderno" es de escaso interés y trata sobre la marginalidad ${ }^{10}$ a pesar de lo cual creemos que podemos entresacar al menos algunas conclusiones y dudas a tener en cuenta:

La identidad sexual y el género si no son lo mismo, son indistinguibles pues se manifiestan de la misma forma y probablemente no hagan referencia a algo anterior a ellos mismos que fuera "natural" y contingente.

El debate planteado por la transexualidad acerca del "sí mismo" sexual (la posibilidad misma de dicho debate) también cuestiona la hipótesis de que el sexo es algo dado.

Definir el sexo en el que una persona debe ser "humanizado" es, en última instancia, una decisión de carácter político y no un acto aséptico de "normalización" o "naturalización"

La "naturaleza" no es algo independiente y anterior a la actividad humana, no tiene un plan, nuestras observaciones de los hechos se ven mediatizados por la existencia de ideas previas que nos hacen encontrar sólo aquello que estamos

\footnotetext{
${ }^{10}$ - ¿cual es tu opinión respecto al postmodernismo?

- creo que es parte de la manera en que la comunidad intelectual realiza su trabajo de marginar y confundir a la gente. No quiero decir que no tenga ningún sentido pero el $90 \%$ es una delusión total (...). Pero cuando miro a esas cositas, se me saltan los ojos, ¿De qué están hablando? (...) Si hay otra cosa que idioteces y trivialidades yo no las he encontrado...

(Chomsky, N., Dieterich, H.; Hablemos de terrorismo. Pamplona: Txalaparta, 1998 citado en Fernández Liria, A., Rodríguez vega, B. La Construcción de narrativas terapéuticas. Desclee de Brouwer. Madrid, 2001)
} 
buscando, nos apoyamos en una idea, un mito para justificar practicas y confundimos los hechos con la tradición.

Nuestro conocimiento actual de la embriología y de la endocrinología nos dice que los machos y las hembras de humanos proceden de embriones con las mismas estructuras. La feminidad y la masculinidad son los extremos de un espectro de múltiples tipos corporales posibles y legítimos.

Reconocer y aceptar la multiplicidad, la riqueza corporal y conductual que expresa la especie humana, y otras (erosdiversidad), es una exigencia de nuestro tiempo, especialmente para la medicina y la psicología.

Tabla 1

Criterios DSM IV-TR para el diagnóstico de F64.x Trastorno de la identidad sexual [302.xx]

A. Identificación acusada y persistente con el otro sexo (no sólo el deseo de obtener las supuestas ventajas relacionadas con las costumbres culturales).

En los niños el trastorno se manifiesta por cuatro o más de los siguientes rasgos:

(1) deseos repetidos de ser, o insistencia en que uno es, del otro sexo

(2) en los niños, preferencia por el transvestismo o por simular vestimenta fe-menina; en las niñas, insistencia en llevar puesta solamente ropa masculina

(3) preferencias marcadas y persistentes por el papel del otro sexo o fantasías referentes a pertenecer al otro sexo

(4) deseo intenso de participar en los juegos y en los pasatiempos propios del otro sexo

(5) preferencia marcada por compañeros del otro sexo

En los adolescentes y adultos la alteración se manifiesta por síntomas tales como un deseo firme de pertenecer al otro sexo, ser considerado como del otro sexo, un deseo de vivir o ser tratado como del otro sexo o la convicción de experimentar las reacciones y las sensaciones típicas del otro sexo.

B. Malestar persistente con el propio sexo o sentimiento de inadecuación con su rol. En los niños la alteración se manifiesta por cualquiera de los siguientes rasgos: en los niños, sentimientos de que el pene o los testículos son horribles o van a desaparecer, de que sería mejor no tener pene o aversión hacia los juegos violentos y rechazo a los juguetes, juegos y actividades propios de los niños; en las niñas, rechazo a or inar en posición sentada, sentimientos de tener o de presentar en el futuro un pene, de no querer poseer pechos ni tener la regla o aversión acentuada hacia la ropa femenina. En los adolescentes y en los adultos la alteración se manifiesta por síntomas como preocupación por eliminar las características sexuales primarias y secundarias (p. ej., pedir tratamiento hormonal, quirúrgico u otros procedimientos para modificar físicamente los rasgos sexuales y de esta manera parecerse al otro sexo) o creer que se ha nacido con el sexo equivocado.

C. La alteración no coexiste con una enfermedad intersexual.

D. La alteración provoca malestar clínicamente significativo o deterioro social, laboral o de otras áreas importantes de la actividad del individuo. 
Figura 1

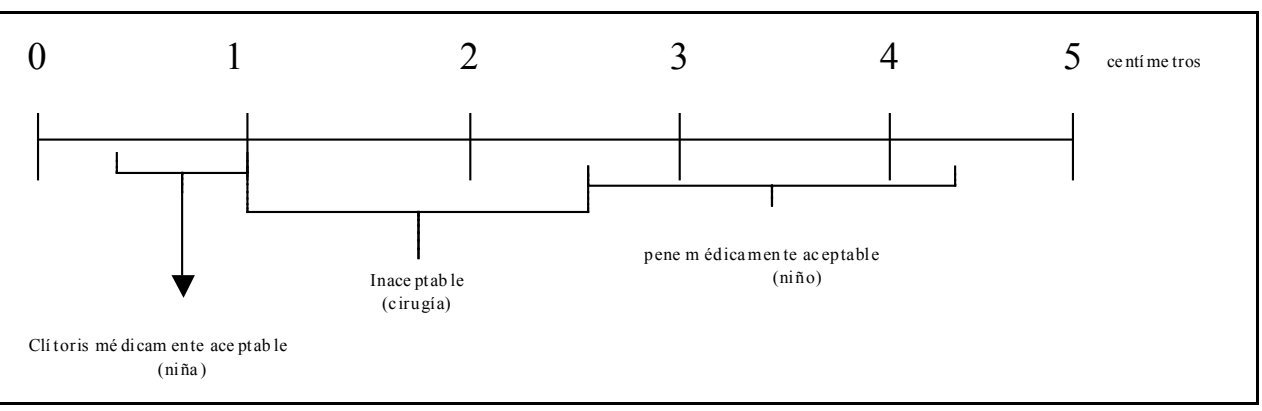

BIBLIOGRAFÍA:

(i) Otero, A. Conducta y patología sexual, en Vallejo Ruiloba, J: Introducción a la psicopatología y a la Psiquiatría(6 ed.). Masson. Barcelona.2006.

(ii) Fausto-Sterling, A. Cuerpos sexuados, Ed. Melusina, Barcelona 2006, pg.144.

(iii) Fausto Sterling, op. Cit.

(iv) Bishop, K.M. y D. WahLsten. 1997. Sex differences in the human corpus callosum: Myth or reality. Neuroscience and biobehavioural reviews 12:581-601.

(v) Fausto-SterLing op.cit.

$\left({ }^{v i}\right)$ Fausto-Sterling, A, op.cit: 232-233.

(vii) Citar trabajos de money

(viii) Stone,S, The empire stryrikes back: a posttransexual manifiesto. Body Guards (1991)

(ix) Benjamín, H, The transexual phenomenon.edición electrónica de Symposion Publishing, Düsseldorf, 1999, originalmente publicado por THE JULIAN PRESS, INC. PUBLISHERS, New York (1966)

${ }^{\mathrm{x}}$ ) Stone,S, The empire stryrikes back: a posttransexual manifiesto. Body Guards (1991)

${ }^{\left({ }^{x i}\right)}$ DSM-IV-TR: manual de diagnostico y estadistico de los trastornos mentales

(xii) CIE 10

(xiii) Preciado, B. Testoyonky, Ed. Espasa Madrid 2008

(xiv) Simone de Beauvoir. El segundo sexo.

${ }^{(x v)}$ Para una explicación de las diferencias entre poder disciplinario y soberano y sus repercusiones en el campo psiquiátrico ver Foucault, M. El poder Psiquiátrico, Akal. 2005 Madrid. Pags.71-97

( ${ }^{\text {xvi }) ~ D e ~ L a u r e n t i s, ~ T . ~ T e c n o l o g i ́ a s ~ d e l ~ g e ́ n e r o . ~}$

(xvii) Fraser, N. ¿de la redistribución al reconocimiento? Dilemas de la justicia en la era "postsocialista”, New Left Review (ed. Española),enero n0, Madrid. 2000.

(xviii) Preciado, B, Devenir bollo-lobo o cómo hacerse un cuerpo queer a partir de El pensamiento heterosexual, en Córdoba,D.,Saez,J. y Vidarte,P.(ed). Teoría Queer. Politicas bolleras, maricas, trans, mestizas..Egales. Editorial Gai y lesbiana. Barcelona. 2001 
ORIGINALES Y REVISIONES

(xix) Monique Wittig. El pensamiento heterosexual.....

${ }^{(\mathrm{xx})}$ Butler, J. El género en disputa. Paidós. Barcelona. 2007

(xxi) Citado en Preciado, B. op.cit.

(xxii) Burgos, E. Hacia la libertad. Contra la violencia. La apuesta de Judith Butler. En García, D. y González, E.(eds.) Entre la ética y la Política. Éticas de la sociedad civil, Publications de la Universitat Jaume I, Collecció e-humanitats,1, Libre electronic,2003.

(xxiii) Preciado, B. op.cit.

(xxiv) Preciado, B. op.cit.

${ }^{\left({ }^{x} v\right)}$ LEY 3/2007, de 15 de marzo, reguladora de la rectificación registral de la mención relativa al sexo de las personas, articulo 4.2.

${ }^{\left({ }^{x x v i}\right)}$ Eribon, D. Reflexiones sobre la cuestión gay. Anagrama. Barcelona.2001.

(xxvii) Fausto-Sterling, A. op.cit.

* MIGUEL HERNÁNDEZ GONZÁLEZ: MIR psiquiatría Hospital Universitario de Canarias. Servicio Canario de la Salud.

GEMMA RODRÍGUEZ MORALES. DUE. Psicóloga. Centro de Salud de la Matanza de Acentejo. Servicio Canario de la Salud.

JOSÉ GARCIA-VALDECASAS CAMPELO. Psiquiatra. Hospital Universitario de Canarias. Servicio Canario de la Salud.

Correspondencia: Correo electrónico: mhdez67@gmail.com

** Recibido: 10/06/2009 